\title{
Searching for the neurobiology of the misinformation effect
}

\author{
Elizabeth F. Loftus \\ University of California-Irvine, Irvine, California 92697, USA
}

For more than 30 years, I have been studying a phenomenon called the misinformation effect (Loftus and Palmer 1974; Loftus 1975; Loftus and Hoffman 1989). The studies that we have done show how readily memory can become skewed when people are fed misinformation. They used a deceptively simple procedure. Subjects first see a complex event, such as a simulated automobile accident. Next, half the subjects receive misleading information about the accident while the others get no misinformation. Finally, all subjects try to remember the original accident. In one actual study using this paradigm, subjects saw an accident and later some of them received misinformation about the traffic sign used to control traffic. The misled subjects got the false suggestion that the stop sign that they had actually seen was a yield sign. When asked later what kind of traffic sign they remembered seeing at the intersection, those who had been given the false suggestion tended to adopt it as their memory, and now claimed that they had seen a yield sign (see Fig. 1). Those who had not received the phony information had much more accurate memories (see Loftus 1979). Taken together, the hundreds, if not thousands, of similar studies show that misinformation can change an individual's recollection in predictable, and sometimes very powerful, ways. In this issue of Learning \& Memory, Okado and Stark (2005) shed light on the neural mechanisms underlying the misinformation effect.

Misinformation can contaminate our memories when we talk to other people, when we are interrogated in a suggestive fashion, or when we see biased media coverage about some event that we may have experienced ourselves. After more than two decades exploring the power of misinformation, we have learned a great deal about the conditions that make people especially susceptible to its damaging influence. We have learned, for example, that memories are more easily modified when the passage of time allows the original memory to fade. Those faded, weakened memories become particularly vulnerable to contamination.

When contemporary investigators began using neuroimaging and other physiological measures to explore true versus false memories, I had hoped that one day these would be applied to the misinformation paradigm. But in misinformation studies, there was often only one critical item (the subject was falsely led to remember a stop sign instead of a yield sign in the accident scene) and thus a maximum of one false memory per subject. Occasionally investigators might try to insert a few false items, but never very many to prevent subjects from catching on to the trick and rejecting the post-event misinformation wholesale.

To do neuroimaging studies of true and false memories, investigators needed to have a decent number of false memories per subject to be able to compare them to true ones. An excellent paradigm for ensuring that a respectable number of false memories were produced was the DRM (after Deese-RoedigerMcDermott, based on work by Deese 1959 and Roediger III and

E-mail eloftus@uci.edu; fax (949) 824-3002.

Article and publication are at http://www.learnmem.org/cgi/doi/10.1101/ Im.90805.
McDermott 1995). In this procedure, subjects study a list of related words such as sour, candy, bitter, sugar, good, taste, tooth, nice, honey, soda, chocolate, cake, heart, tart, and pie. When later tested, subjects often think they heard or saw certain non-presented items (sweet, in this case). The procedure reliably led most subjects to produce a goodly number of false memories; that is, subjects would describe the falsely remembered word in detail, and gave every indication that they were remembering a real event.

Thus, virtually all of the neuroimaging studies of true and false memories used the DRM paradigm, or some variant (e.g., Cabeza et al. 2001). Therefore, most of what we learned about the neurobiology of true and false memories was about the neurobiology of true and false memories of words, or at least about memories that were relatively pallid in nature.

Enter the work of Okado and Stark (2005). First, and importantly, they developed materials that were far closer to the complex visual scenarios used in most of the misinformation research. Moreover, their procedure was one in which a decent number of false memories could be produced in a given subject, thereby allowing meaningful comparisons with the true memories. And in the process they have taught us something about the misinformation effect that can relate to the hundreds of behavioral studies that have been done on the topic.

Okado and Stark (2005) used eight complex vignettes each of which were shown to participants through 50 color slides. One vignette, for example, depicted a man stealing a girl's wallet and subsequently trying to hide. Thus, the materials were far more complex and "realistic" than had been used in the typical neuroimaging memory study. Subjects were scanned while they watched these original vignettes. Later, during the misinformation phase, subjects watched the vignettes again, under the belief that they were watching the same scenes. However, 12 critical slides were altered in some way. For example, if the original event showed the man hiding behind a door, the misinformation slide showed him hiding behind a tree.

Two days later, subjects took a recognition test on their memory for the original scenarios. It included questions such as "Where was the man hiding after he stole the girl's wallet and crossed the street? Behind a door? Behind a tree? Behind a car?"1 On a subsequent "source memory" test, subjects indicated the source of their memory for every answer they had given during the first recognition test. Thus, if they claimed on the recognition test that the man was hiding behind a tree, they would indicate that they knew this because they saw it in the original presentations, they saw it in the second set of presentations, they saw it in both, they noticed conflict, or they simply guessed. A misinformation error was observed whenever subjects said they saw the misinformation item (hiding behind a tree) during the original presentation (whether only then, or whether they said they saw this in both presentations).

${ }^{1} \mathrm{~A}$ few other examples of questions used in the research were "After the man bumped into her, what part of the girl's body was hurt? Her neck? Her arm? Her back?" "What did the man, in frustration, do to the car after he slammed the trunk on his hand(s)? Kick the car? Hit the car? Scratch the car?" 

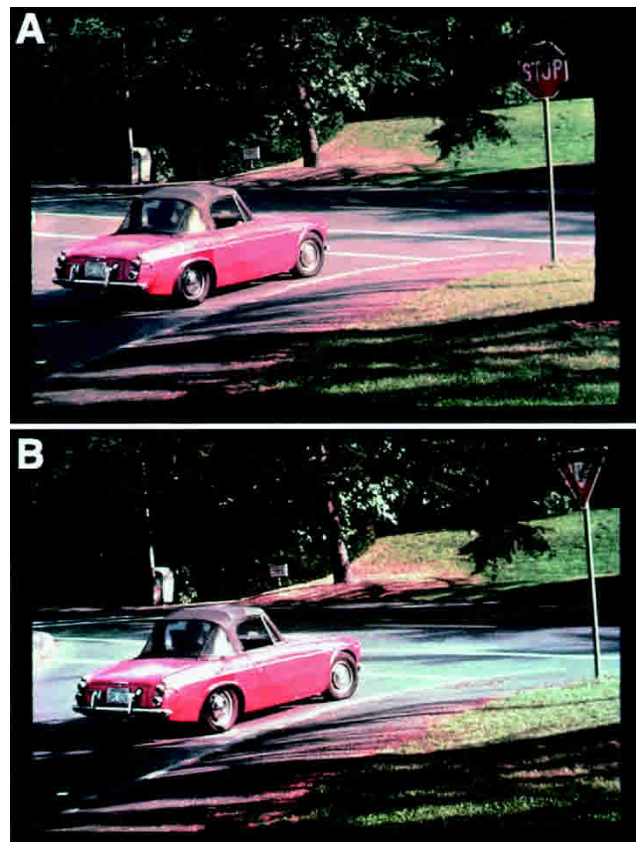

Figure 1. Images used in a classic misinformation experiment performed by Loftus et al. (1978). (A) Traffic intersection with stop sign. (B) Misinformation image with stop sign replaced by yield sign. A detailed account of the research can be found in Loftus 1979.

How often did subjects make misinformation errors? First, subjects endorsed the misinformation items quite often on the recognition test, and significantly more often than they endorsed ordinary foil items. In our example, they claimed the man hid behind a tree. This standard misinformation effect was, of course, entirely expected. Of course, some of these endorsements might not be clear cases of false memories. After all, on subsequent source memory tests, subjects occasionally classified misinformation responses as "guesses." When subjects claimed to have recognized the misinformation item, they typically said that they remembered the item only from the original event ( $27 \%$ of the time) or that they saw the item in both events $(20 \%$ of the time). Thus, the pictorial version of the misinformation paradigm readily created false memories.

The major contribution of the current work is that the neural activity during the encoding of the original presentations and the misinformation presentations was predictive of whether a misinformation error would occur. Activity in particular brain regions such as the left hippocampus predicted whether the critical items from the original presentations or misinformation presentations were later remembered on the recognition test. The reported misinformation items were robust false memories, ones misattributed to the original event during the source test, and activity in other brain regions such as the prefrontal cortex correspondingly showed that for these misinformation errors, context/source encoding was strong during the original event phase and poor during the misinformation phase, which set up a vulnerable situation whereby the misinformation item was easily embedded into the context of the original event, creating strong false memories.

These findings are important in their own right, but they also dovetail nicely with behavioral discussions of the misinfor- mation effect. Earlier we proposed a discrepancy detection model to discuss various misinformation results (Loftus 1991). The idea is that people are likely to reject misinformation and produce the original true event item when they notice a discrepancy at the time the misinformation is processed. Any variables that lower the likelihood that a discrepancy is detected should reduce the misinformation effect. Therefore, shorter exposure time of the original event, longer retention intervals between the original and misinformation phases, and warnings about potential misinformation prior to the misinformation phase all reduced the misinformation effect, consistent with the notion of discrepancy detection. The current neuroimaging results, and especially patterns of activity seen during the misinformation phase, are consistent with these discrepancy notions. The neuroimaging gives us a window into that misinformation processing that before now we could only speculate about.

While the specific neuroimaging results reported by Okado and Stark (2005) are highly detailed, complex, and well discussed by them in their paper, let it suffice to say that they have provided here the first research to date that uses neuroimaging to reveal underlying mechanisms of the misinformation effect. For more than a half century, investigators have been actively interested in relating the brain to memory (Hebb 1949; Hull 1952), an enterprise that has grown far more rapidly than even they might have foreseen (Cahill et al. 2001). Okado and Stark's (2005) innovations in determining an elegant way to apply neuroimaging to an important memory problem would make their forebears proud. It will be a long time before these results have practical use, for example, in discriminating whether a particular memory report derives from an original event or from misinformation (something the judicial system might like to see), but the current investigation is certainly an important first step.

\section{References}

Cabeza, R., Rao, S.M., Wagner, A.D., Mayer, A.R., and Schacter, D.L. 2001. Can medial temporal lobe regions distinguish true from false? An event-related functional MRI study of veridical and illusory recognition memory. Proc. Natl. Acad. Sci. 98: 4805-4810.

Cahill, L., McGaugh, J.L, and Weinberger, N.M. 2001. Trends Neurosci. The neurobiology of learning and memory: Some reminders to remember. 24: 578-581.

Deese, J. 1959. On the prediction of occurrence of particular verbal intrusions in immediate recall. J. Exp. Psychol. 58: 17-22.

Hebb, D.O. 1949. The organization of behavior. Wiley, New York.

Hull, C.L. 1952. A behavior system. Yale University Press, New Haven, CT.

Loftus, E.F. 1975. Leading questions and the eyewitness report. Cognit. Psychol. 7: 560-572.

. 1979. Eyewitness testimony. Harvard University Press, Cambridge, MA.

. 1991. Made in memory: Distortions in recollection after misleading information. Psychol. Learn. Motiv. 27: 187-215.

Loftus, E.F. and Hoffman, H.G. 1989. Misinformation and memory: The creation of memory. J. Exp. Psychol. General 118: 100-104.

Loftus, E.F. and Palmer, J.P. 1974. Reconstruction of automobile destruction: An example of the interaction between language and memory. J. Verbal Learn. Verb. Behav. 13: 585-589.

Loftus, E.F., Miller, D.G., and Burns, H.J. 1978. Semantic integration of verbal information into a visual memory. J. Exp. Psychol. Hum. Learn. 1: $19-31$.

Okado, Y. and Stark, C.E.L. 2005. Neural activity during encoding predicts false memories created by misinformation. Learn. Mem. (this issue).

Roediger III, H.L. and McDermott, K.B. 1995. Creating false memories: Remembering words not presented in lists. J. Exp. Psychol. Learn. Mem. Cogn. 21: 803-814. 